\title{
Organic Light Emitting Devices with Nano-ZnO Thin Films as Cathode Buffer Layer
}

\author{
Yinglian Wang, Junyao Ye \\ Department of Mechanical and Electronic Engineering, Jingdezhen Ceramic Institute, Jiangxi, China \\ sdwyl@126.com
}

Keywords: Organic light emitting device; $\mathrm{ZnO}$ cathode buffer layer; vacuum thermal evaporation; current density; luminous efficiency

Abstract: Organic light emitting devices (OLED) with the structure of ITO / TPD / $\mathrm{Alq}_{3} / \mathrm{ZnO} / \mathrm{Al}$ were prepared by vacuum thermal evaporation method. By establishing multi-layer structure model, we discussed the influence of $\mathrm{Alq}_{3}$ deposition thickness and $\mathrm{ZnO}$ buffer layer on the photoelectric performance of the device, got the relationship of current density and work voltage. The results show that $\mathrm{ZnO}$ buffer layer can produce additional dipole energy and reduce the electronic potential barrier of cathode, its best thickness is $3 \mathrm{~nm}$, too thin or too thick will increase the driving voltage, block carriers injected. When the thickness of $\mathrm{Alq}_{3}$ film is $50 \mathrm{~nm}$, the photoelectric performance is best, current density reaches $100 \mathrm{~mA} / \mathrm{cm}^{2}$, the increase of $\mathrm{Alq}_{3}$ thickness will make the electronics through the layer become scarce, affect the compound with holes. The results provide theoretical guidance to optimize the design and increase the performance of OLED.

\section{Introduction}

OLED with its superior performance has become the most popular research of display device, as a new electroluminescent devices, its simple structure, low cost, suitable for wide production [1]. Bell Labs' Dodabalapar [2] and co-workers used micro-cavity structure to improve the luminescence properties of the device, this structure can also change the color of light-emitting devices, or even to achieve color display. L. S. Liao [3] achieved a series structure connected with organic material, which not only greatly improves the efficiency, but also can improve the life of the device. All kinds of attempts were in order to improve its electro-optical properties, reduce driving voltage, improve luminous efficiency and prolong service time. Such as the ITO surface treatment, the film annealing treatment, the buffer layer injection, superlattice structure and so on, each method has different degrees improvement of OLED photoelectric performance. Comparing the different materials used for cathode buffer layers, metal oxide is better than the high function metal and organic materials to the performance of organic light emitting diodes, especially the improvement of stability and service life. The forbidden band width of most metal oxide is above $3 \mathrm{eV}$, at normal temperature, carriers concentration is low, resistivity is big, is generally insulator. At present, some metal oxide semiconductor materials such as $\mathrm{TiO} 2$ [4] and $\mathrm{MoO} 3$ thin films have been used as injection layer successfully, the start voltage reduced to 3-4V, brightness and efficiency have been greatly enhanced. $\mathrm{ZnO}$ semiconductor thin film has high transmissivity, wide forbidden band (3.2ev), good physical properties and chemical stability, is widely used in solar cells and many kinds of optical thin film technologies [5]. In this paper, OLED with the structure of ITO / TPD / $\mathrm{Alq} 3$ / $\mathrm{ZnO} / \mathrm{Al}$ have been prepared by vacuum evaporation coating method. The influence of Alq3 thickness and $\mathrm{ZnO}$ buffer layer to the photoelectric performance of OLED has also been observed. 


\section{Materials and Methods}

Using the conductive ITO glass with the square resistance $20 \Omega$ as anode, in turn, etching, alcohol cleaning, chromium acid soaking and deionized water ultrasonic cleaning, and the last drying in nitrogen atmosphere. Use vacuum evaporation coating method, keep the bottom vacuum for $3 \times 10^{-4} \mathrm{~Pa}$, TPD coating thickness is $30 \mathrm{~nm}$ as the hole-transport layer, evaporating Alq3 as luminous layer and $\mathrm{ZnO}$ as buffer layer with different thickness, at last $\mathrm{Al}$ as cathode, constitute the typical structure ITO/TPD/Alq3/ZnO/Al of organic light-emitting device.

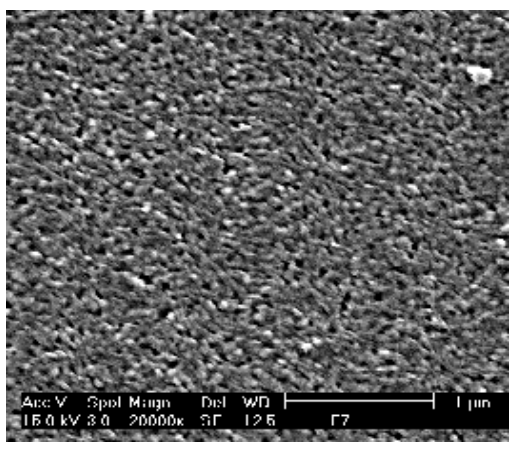

(a)

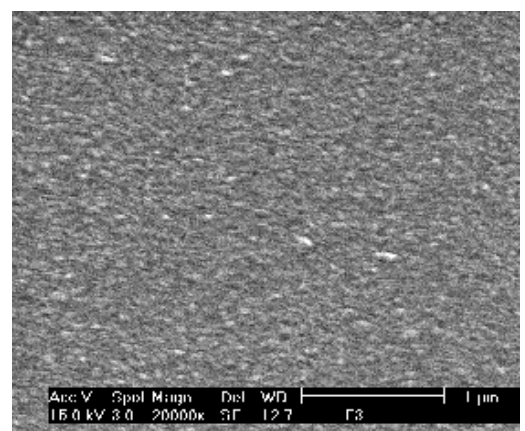

(b)

Fig.1. (a) SEM of Alq3 (b) SEM of Alq3 coated by ZnO

The influence of Alq3 surface morphology to the photoelectric performance of OLED is not ignored. Too many defects will become carriers capture traps, affect the luminous efficiency. From fig. 1 we can see Alq3 surface is rough with many pores, this is because the vacuum chamber residual gas adsorption in the film in the process of composition, so the film structure is loose. When $\mathrm{ZnO}$ layer covered, the surface defect decrease, uniform structure, can block the spread of Al to Alq3 layer, reduce the interface potential barrier, improving the electro-optical property.

\section{Results and Discussion}

The curves have typical p-n rectifier characteristics, and in ITO / TPD / Alq3 / Al structure study found similar rectifier features. Compared with no $\mathrm{ZnO}$ buffer layer, when its thickness less than $2.0 \mathrm{~nm}$, the current density and voltage curve (that is J-V curve) bending to high voltage, which shows that there is great resistance between cathode and organic layer of the structure, to a certain extent, it is caused by the no idealized preparation process of the samples. In addition, interface dipole energy almost no change, at the same time also have no potential barrier reduce. Predictably, too thin $\mathrm{ZnO}$ layer will reduce the light conversion efficiency. However, with the further increase of $\mathrm{ZnO}$ thickness, carriers injection significantly increased. When $\mathrm{ZnO}$ thickness is greater than $2.0 \mathrm{~nm}$, relative to no insert $\mathrm{ZnO}$ devices, its $\mathrm{J}-\mathrm{V}$ curve rise faster, that is to say, in the same driving voltage, the current density would be much higher than no $\mathrm{ZnO}$ layer devices, so that the electronic injection is be strengthen. As previously assumption, the holes injection is constant, the carriers injection towards balance gradually. This is caused by the reduction of the relative potential barrier between Fermi level EF energy of cathode and the organic layer LUMO level. This may be because of the rise of EF energy of cathode or the reduction of interface energy gap, the interface resistance is reduced many [6]. This may be because $\mathrm{ZnO}$ molecular filling in the interface pore, make $\mathrm{Al}$ and Alq3 in the form of better contact on the surface, which reduces the potential barrier. We believe that the underlying factor of the impact of electronic injection and $\mathrm{J}-\mathrm{V}$ curve is the additional dipole energy produced by $\mathrm{ZnO}$ thin film. At the same time too 
thick layer of $\mathrm{ZnO}$ can make additional resistance, may discourage carrier injection and increase the driving voltage. Therefore, $3 \mathrm{~nm} \mathrm{ZnO}$ buffer layer is the best thickness for cathode.

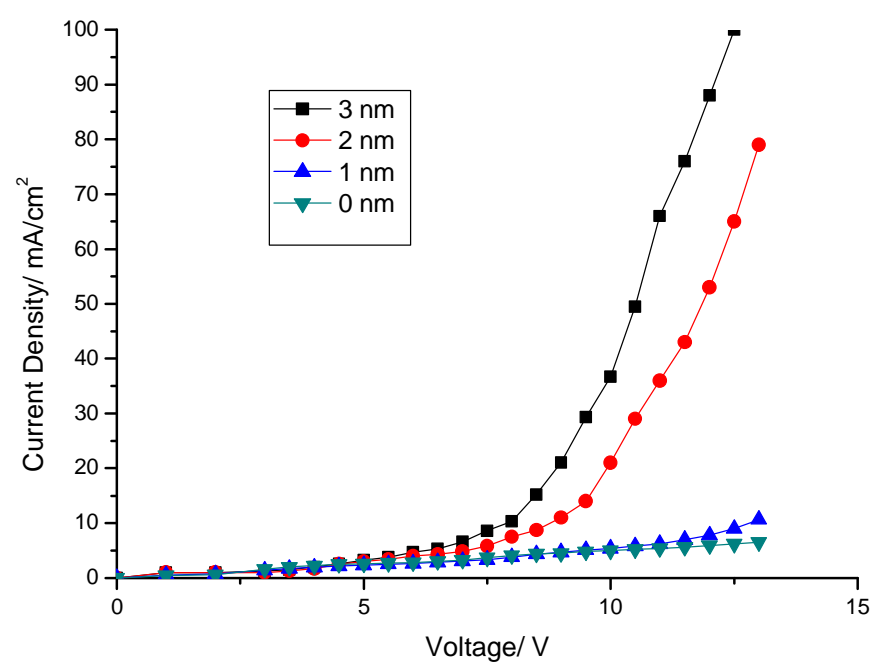

Fig.2. The J-V curves of different $\mathrm{ZnO}$ thickness of OLED

Fig. 3 is the $\mathrm{J}-\mathrm{V}$ curves of different Alq3 thickness OLED and the $\mathrm{ZnO}$ buffer layers are all $3 \mathrm{~nm}$. It shows that the driving voltage increase with increased Alq3 thickness. From $J=\sigma E$ we can see the field strength abate with the increase of Alq3 layer thickness, current also. On the other hand, the increased Alq3 layer thickness will hinder electronic injection, restrain the composite of electronic and hole from HTL layer at the Alq3 / TPD interface. At this time the current in Alq3 weaken, the device performance becomes poor. According to the literature [7] are elaborated, when no $\mathrm{ZnO}$ buffer layer, with the driving voltage function, electronic must tunneling through the triangle barrier. $\mathrm{ZnO}$ buffer layer has two effects: (1) It's voltage drop equal to the relatively improved cathode EF energy in the value, and reducing the triangle barrier in Alq3 layer. (2) Buffer layer produced an additional potential barrier by itself.

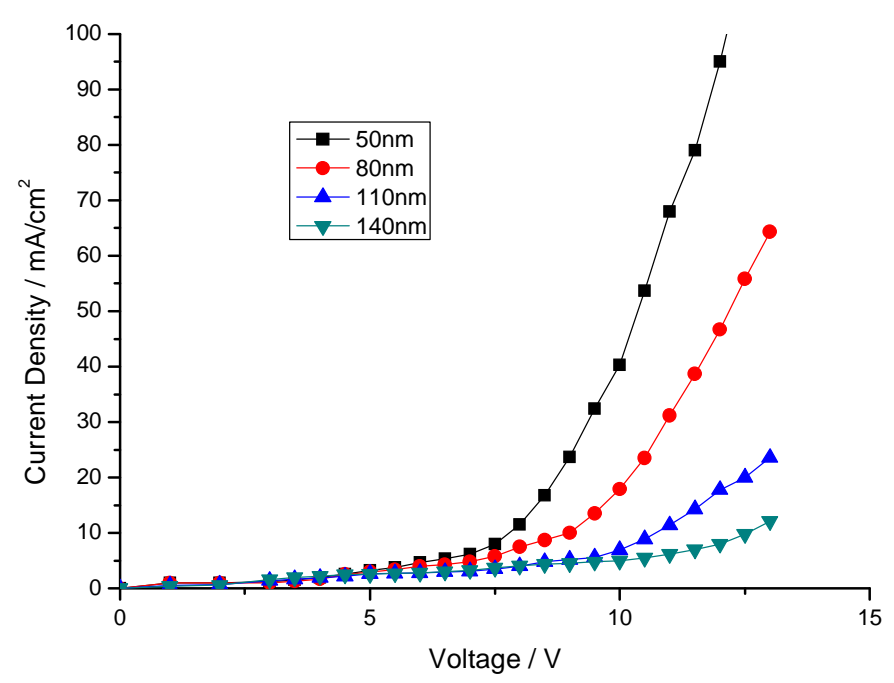

Fig.3. The J-V curves of different Alq3 thickness of OLED 
Compared with the normal device with $\mathrm{Al}$ cathode, the efficiency and brightness of the devices with $\mathrm{ZnO}$ as cathode have been effectively enhanced, and the power efficiency of the latter has been improved to be about 1.15 times. We think the incorporation of the $\mathrm{ZnO}$ layer will effectively enhance the electron injection and the electrons and the holes will be balanced in the emitting layer. Further more, the buffer layer will block the diffusion of the Al atoms to Alq3 layer and thus decrease the quenching centers [8].

\section{Conclusion}

OLED with the structure of ITO / TPD / Alq3 / ZnO / Al were prepared by vacuum evaporation coating method. By establishing multi-layer structure model, discussed the influence of Alq3 deposition thickness and $\mathrm{ZnO}$ buffer layer on the photoelectric performance of the device, got the relationship of current density and work voltage. We found $\mathrm{ZnO}$ can significantly improve the performance of OLED, this is produced by $\mathrm{ZnO}$ additional dipole energy, it can reduce the electronic potential barrier at the cathode, so as to improve the performance of OLED. The best thickness of $\mathrm{ZnO}$ buffer layer is $3 \mathrm{~nm}$, too thin or too thick will increase the driving voltage, block carriers injection. The increase thickness of Alq3 layer will lead to few electronic through the layer, restrain the composite of electronic and the hole from hole-transport layer at Alq3 / TPD interface.

\section{References}

[1] C. W. Tang, S. A. Vanslyke, C. H. Chen. Electroluminescence of doped organic thin films. Journal of Applied Physics, 1989, 65(9):3610-3616

[2] A. Dodabalapur, R. H. Jordan,M. Strukelj, T. M. Miller, White organic electroluminescence devices, Appl. Phys. Lett. (1996) 68, 1192

[3] L. S. Liao, G. Cheng, Y. F Zhang, et al. White organic light-emitting devices with a phosphorescent multiple emissive layer, Appl.Phys. Lett. (2006) 89, 043504

[4] Zhang Z F, Deng Z B, Liang C J. Organic light-emitting diodes with a nano structured TiO2 layer at the inter face between ITO and NPB layers. Displays, 2003, 24: 231-2341

[5] Z. Wu, S. Chen, H. Yang, et al. Top-Emitting Organic Light-Emitting Devices Based on Silicon Substrate with High Luminance and Low Turn-on Voltage, Chin. Phys. Lett. 2005, 22:233

[6] Zhao J M, Zhan Y Q, Zhang S T. Mechanisms of injection enhancement in organic light-emitting diodes through insulating buffer. Appl.Phys.Lett. 2004,84:5377-5379

[7] C B Lee, A Uddin, X Hu, T G Andersson. Study of Alq3 thermal evaporation rate effects on the OLED. Materials Science \& Engineeting B. 2004,112:14-18

[8] S Besbes, A Ltaief, K Reybier, L Ponsonnet, N Jaffrezicc, Davenas, H. Ben Ouada. Injection modifications by ITO functionalization with a self-assembled monolayer in OLEDs. Synth. Met. 2008,138:197-200 